\section{Relationship between the Number of Interacting Particles and Flux Ratio}

WhEN the flux ratio does not obey the Ussing relationship $^{1}$ in a passive system, interaction must be occurring between molecules of substrate passing through the mem. brane. By using a thermodynamic argument based on inducod transport ${ }^{2}$ it is possible to relate the deviation from the Ussing relationship to the minimum number of particles that interact without reference to the mechanism involved.

Consider two chambers I and II separated by a passive membrane. Suppose that the concentration of the substrate in question is $C_{\mathrm{I}}$ in chamber $\mathrm{I}$ and $C_{\mathrm{II}}$ in chamber II, and that there is a net flux of substrate from I to II. Let a very small quantity of radioactive substrate be added to the chambers. As diffusion of the substrate through the membrane occurs, suppose that there is a device that selectively removes the non-radioactive molecules of substrate from one chamber and returns them to the other so that the concentrations $C_{\mathrm{I}}$ and $C_{\mathrm{II}}$ are maintained unchanged. As a consequence of this process the radioactive molecules will eventually become distributed in such a manner that the flux of radioactive molecules in the two directions are equal, or:

$$
k_{\mathrm{I}} m_{\mathrm{I} \rightarrow \mathrm{II}}=k_{\mathrm{II}} m_{\mathrm{II} \rightarrow \mathrm{I}}
$$

where $k_{\mathrm{I}}$ is the specific activity of the substrate in cham. ber $I, k_{\text {II }}$ is the specific activity of the substrate in chamber II, $m_{I \rightarrow I I}$ is the flux of substrate from chamber I to II, and $m_{I I \rightarrow I}$ is the flux of substrate from chamber II to I.

As equilibrium is approached the free energy required to transport $\Delta m^{\prime}$ moles of radioactive substrate from chamber I to II becomes:

$$
\left(R T \ln \frac{f_{\mathrm{II}} C_{\mathrm{II}} k_{\mathrm{II}}}{f_{\mathrm{I}} C_{\mathrm{I}} k_{\mathrm{I}}}-z E F\right) \Delta m^{\prime}
$$

where $E$ is the electrical potential difference between chamber I and II ( $E$ positive if chamber $I$ is positive), $z$ is the number of positive electrical charges on one molecule of substrate, $f_{\mathrm{I}}$ is the activity coefficient of substrate in chamber I, $f_{\mathrm{II}}$ is the activity coefficient of substrate in chamber II, $R$ is the gas constant and $T$ is the absolute temperature. This expression is derived on the assumption that the energy associated with radioactive and non-radioactive molecules is the same, and from a consideration of the entropy changes based on the probabilities of the systems. The free energy made available when $\Delta m$ moles of non-radioactive substrate pass from chamber I to II is, on the other hand:

$$
\left(R T \ln \frac{f_{\mathrm{I}} C_{\mathrm{I}}}{f_{\mathrm{II}} C_{\mathrm{II}}}+z E F\right) \Delta m
$$

Since the energy to transport the radioactive substrate molecules must have come from the movement of nonradioactive substrate, it follows that if $\Delta m$ molecules of non-radioactive substrate transport $\Delta m^{\prime}$ moleculos of radioactive substrate from chambers I to II :

$$
\begin{aligned}
& \left(R T \ln \frac{f_{\mathrm{I}} C_{\mathrm{I}}}{f_{\mathrm{II}} C_{\mathrm{II}}}+z E F\right) \Delta m> \\
& \left(R T \ln \frac{f_{\mathrm{II}} C_{\mathrm{II}} k_{\mathrm{II}}}{f_{\mathrm{I}} C_{\mathrm{I}} k_{\mathrm{I}}}-z E F\right) \Delta m^{\prime}
\end{aligned}
$$

$$
\text { Let } r=\frac{\Delta m+\Delta m^{\prime}}{\Delta m^{\prime}} \text {, then from equations (1) and (4): }
$$

$$
\frac{m_{\mathrm{I} \rightarrow \mathrm{II}}}{m_{\mathrm{II} \rightarrow \mathrm{I}}} \gtrless\left(\frac{f_{\mathrm{I}} C_{\mathrm{I}}}{f_{\mathrm{II}} C_{\mathrm{II}}} \mathrm{e}^{z E F / R T}\right)^{r}
$$

Consider the process involved in the interaction (for example, a polyvalent carrier or a long pore). If one radioactive substrate molecule takes part at any instant in the process it is probable that all the other substrate molecules at the same instant are non-radioactive since the radioactive molecules are present in extremely small concentration. Hence $r$ must represent the number of molecules that, on average, interact. Consequently, if an equality is assumed in equation (5) the experimental value of $r$ will give the minimum number of interacting particles.

It is possible for the interaction to be such that the radioactive substrate molecules are induced to travel in the opposite direction to the movement of non-radioactive molecules. In this case, equation (2) has a negative sign and if $\Delta m / \Delta m^{\prime}=r^{\prime}$ :

$$
\frac{m_{\mathrm{I} \rightarrow 1 \mathrm{I}}}{m_{\mathrm{II} \rightarrow \mathrm{I}}}>\left(\frac{f_{\mathrm{I}} C_{\mathrm{I}}}{f_{\mathrm{II}} C_{\mathrm{II}}} \mathrm{e}^{z E F / R T}\right)^{1-r^{\prime}}
$$

$r^{\prime}$ is the average number of non-radioactive molecules associated with the movement of one radioactive molecule in the opposite direction. Again, if an equality is assumed in equation (6) the experimental value of $r^{\prime}$ will represent the minimum number of particles.

The flux ratio criterion may be applied to chemical reactions ${ }^{3}$ and similar arguments may also be used concerning the number of interacting particles.

\section{H. G. Britton}

Department of Physiology,

St. Mary's Hospital Medical School, London, W.2.

1 Ussing, H. H., Acta Physiol. Scand., 19, 43 (1949).

Britton, H. G., Nature, 198, 190 (1963).

${ }^{3}$ Britton, H. G., Nature, 205, 1323 (1965).

\section{Use of Acidic Degradation for the Determination of the Distribution of Pyrimidine Nucleotides in Deoxyribonucleic Acids}

Acroic degradation has been widely used to determine the distribution of the pyrimidine nucleotides in deoxyribonucleic acids (DNA; I). Two main methods have been used; in one the DNA was treated with $0.1 \mathrm{M}$ sulphuric acid at $100^{\circ}$ for $35 \mathrm{~min}$, and in the other, 67 per cent formic acid and 2 per cent diphenylamine at $30^{\circ}$ for $17 \mathrm{~h}$ were used $^{2}$. In both procedures it appears that the first stage is the hydrolysis of the purine glycosidic linkages to give apurinic acid (II).

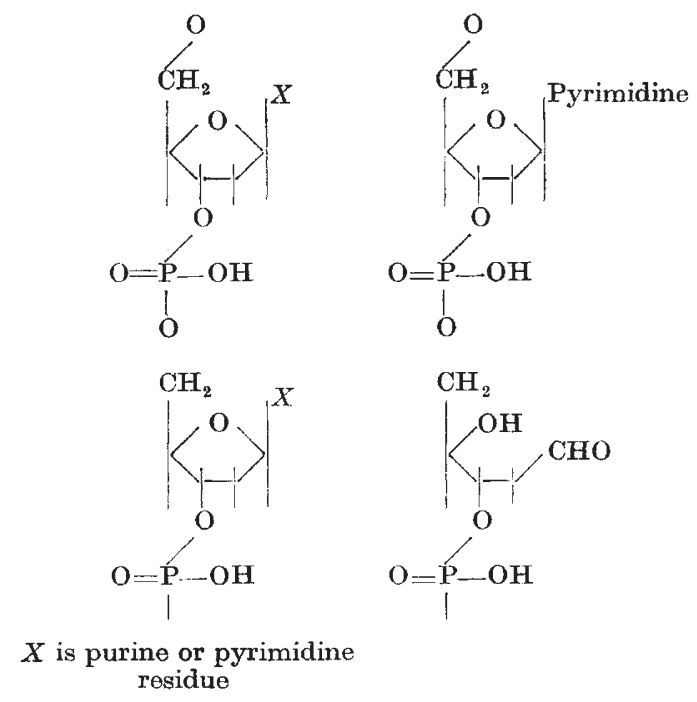

(I)

It is claimed by the authors of the two methods that the next stage is the degradation of the apurinic acid to give products with the structure (pyrimidine nucleoside) $n$ phosphate $_{n+1}$ (III). 